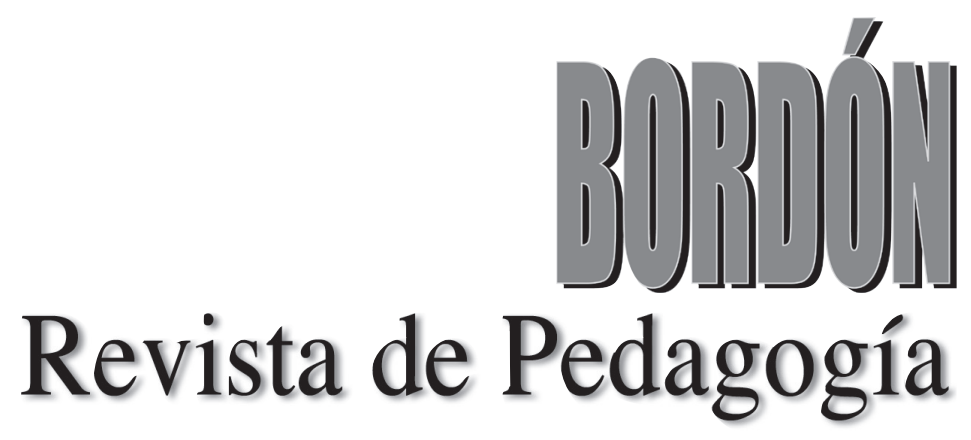

Volumen 69

Número, 2

2017

SOCIEDAD ESPAÑOLA DE PEDAGOGía 


\title{
LA CULTURA DE PAZ En EDUCACIÓn SECUNDARIF OBLIGATORIA. ESTUDIO COMPARADO DE LIBROS DE TEXTO DE EDUCACIÓn PARA LA CIUDADAnÍ́
}

\section{Teaching the culture of peace at the level of Compulsory Secondary Educational. A comparative study of textbooks on Citizenship Education}

\author{
SEBASTIÁN SÁNCHEZ FERNÁNDEZY MIRIAM VARGAS SÁNCHEZ \\ Universidad de Granada
}

DOI: 10.13042/Bordon.2016.49768

Fecha de recepción: 29/04/2016 - Fecha de aceptación: 21/12/2016

Autor de contacto / Corresponding Author: Sebastián Sánchez Fernández. E-mail: ssanchez@ugr.es

Fecha de publicación online: 27/01/2017

INTRODUCCIÓN. Esta investigación tiene como objetivo fundamental conocer y analizar la presencia y el tratamiento didáctico de los contenidos relacionados con la educación para la cultura de paz en los libros de texto de Educación para la Ciudadanía en Educación Secundaria Obligatoria, por tratarse de la materia más cercana a estos contenidos. MÉTODO. Revisado el estado de la cuestión, se han analizado desde un enfoque cualitativo seis libros de texto de Educación para la Ciudadanía en Educación Secundaria Obligatoria. Cada libro se ha estudiado a través de seis indicadores elaborados teniendo en cuenta los principales documentos que explican y desarrollan la cultura de paz. Para la valoración de la presencia y el tratamiento de estos indicadores hemos establecido como criterios la combinación de dos variables, la profundidad y la extensión, con tres grados de valoración para cada una de ellas: ausente, superficial y profundo, para la primera; y ausente, breve y amplio, para la segunda. RESULTADOS. Hemos detectado que la mayoría de los libros de texto analizados recogen y desarrollan aceptablemente casi todos los contenidos reflejados en los indicadores utilizados, además de denunciar todos ellos la violencia existente y de hacer propuestas de construcción de una cultura de la paz a diferentes niveles y en distintos ámbitos, desde el internacional a la propia cotidianeidad. DISCUSIÓN. Contrastando nuestros resultados con otros estudios y con documentos legislativos en educación, hemos comprobado que la desaparición de la asignatura Educación para la Ciudadanía supone un importante freno para la formación en los valores fundamentales relacionados con la cultura de paz, especialmente importantes en esta etapa educativa.

Palabras clave: Paz, Educación para la Ciudadanía, Educación Secundaria Obligatoria, Libros de texto, Educación en Valores. 


\section{Introducción}

La UNESCO a partir de 1948 ha ido incorporando los llamados Derechos de Tercera Generación ${ }^{1}$, entre los que destaca el derecho a la paz, como derecho a vivir en paz, al respeto a la vida y a una vida digna. En documentos más recientes $^{2}$, se destaca que la educación es la clave para llevar a cabo un proyecto común para la construcción de la paz y el progreso de los pueblos, planteando como gran desafío invertir concienzudamente en una educación para todos, durante toda la vida.

La primera definición de cultura de paz se realizó en el Congreso Internacional de Yamoussoukro, Costa de Marfil, en 1989. Posteriormente, la Asamblea General de Naciones Unidas ${ }^{3}$ la definió como un "conjunto de valores, actitudes, tradiciones, comportamientos y estilos de vida" basados en una serie de principios y compromisos, reconociendo expresamente que "la educación a todos los niveles es uno de los medios fundamentales para edificar una cultura de paz" (art. 4).

Siguiendo las Recomendaciones del Consejo de Europa $^{4}$, en las que se instaba a los Estados miembros a desarrollar políticas educativas en consonancia con la educación para la ciudadanía democrática, en el año 2006 en España, la Ley Orgánica de Educación (LOE) incorpora la asignatura Educación para la Ciudadanía ${ }^{5}$, dando pie a la publicación de los primeros libros de texto de esta asignatura, con el objetivo de promover la práctica de la democracia y la formación en valores cívicos en el alumnado.

En torno a la visión de la asignatura de Educación para la Ciudadanía en la prensa, García López y Murillo (2009) señalan que la prensa está más interesada en destacar las repercusiones externas que ha provocado la asignatura que en informar a la población sobre los contenidos de la misma.

El cambio de Gobierno producido tras las elecciones generales de noviembre de 2011, junto con la amplia polémica suscitada en torno a la materia, conlleva que en 2013 se adopte la eliminación de la asignatura de Educación para la Ciudadanía, como consecuencia de la aprobación de la Ley Orgánica para la Mejora de la Calidad Educativa (LOMCE), cuyo calendario de implantación permite que esta asignatura siga vigente en la Educación Secundaria Obligatoria durante el actual curso 2015-2016.

En este trabajo nos interesa conocer y analizar la presencia y el tratamiento de los contenidos relacionados con la cultura de paz en los libros de texto de Educación para la Ciudadanía en la Educación Secundaria Obligatoria, al ser esta la materia más próxima y afín en la que podemos encontrar temáticas favorecedoras de una cultura de la paz en la educación, especialmente en las etapas obligatorias del sistema educativo.

En estrecha relación con el concepto de cultura de paz, Martínez Guzmán (2000) plantea su filosofía para hacer las paces, desde la que propone adquirir un compromiso con el fomento de las capacidades, posibilidades y competencias para deconstruir y desaprender la violencia. Tenemos criterios para reconocer y denunciar la guerra, la marginación y la exclusión, por lo que también podemos aprender a hacer las paces.

Generalmente no solemos prestar atención a las manifestaciones de paz que se dan en nuestra vida diaria, y no es porque no se produzcan, sino porque ponemos más la mirada en la violencia, aunque sean más habituales las relaciones de convivencia pacífica en los diferentes ámbitos de la cotidianidad, especialmente en los escolares (Sánchez Fernández y Sánchez Vázquez, 2012). Conocer y denunciar cómo se articulan y retroalimentan los distintos tipos de violencia (directa, estructural, cultural, etc.) es absolutamente necesario para poder hacerle frente, buscando su disminución y su erradicación; pero también es preciso superar los enfoques centrados en la violencia para buscar la transformación de una cultura de la violencia hacia una cultura de la paz, en la línea que proponen 
Naciones Unidas (UNESCO, 1996) y relevantes aportaciones de la investigación para la paz (Muñoz, 2001).

\section{Objetivos}

Los objetivos de este trabajo son:

- Analizar cómo aparecen y se tratan los contenidos de la cultura de paz en seis libros de texto de Educación para la Ciudadanía de Educación Secundaria Obligatoria.

- Visibilizar y analizar las manifestaciones de la cultura de paz encontradas en los libros de texto estudiados.

\section{Método}

Hemos utilizado una metodología de análisis cualitativo partiendo de las limitaciones que plantean Boqué, Alguacil, Pañellas, García Raga y Rosich (2013), al poner de manifiesto que no existen aún medidas o indicadores con los que medir la paz, con las dificultades que ello genera. Por su parte, De Rivera (2004) plantea que los indicadores más importantes para una cultura de paz en la sociedad actual según la UNESCO son los siguientes: educación para la paz y la resolución de conflictos; desarrollo sostenible; derechos humanos; igualdad de género; participación democrática; entendimiento, tolerancia y solidaridad; comunicación participativa y libre flujo de información; y paz internacional y seguridad. En nuestro caso, al no poder contar con indicadores y ámbitos concretos para poder ser aplicados en el análisis de los libros de texto, hemos optado por la creación de unos indicadores que nos permitieran abordar el estudio de la manera más coherente posible con nuestros objetivos. Para ello hemos partido de las propuestas recogidas en los documentos que cuentan con mayor consenso internacional en la conceptualización y el desarrollo de la cultura de paz: la Resolución 53/243 de la Asamblea
General de Naciones Unidas ${ }^{6}$ y el Manifiesto $2000^{7}$. Como resultado se han configurado los siguientes seis ámbitos de contenidos de la cultura de paz, donde se incluyen los aspectos más representativos de ambos documentos, que utilizamos a modo de indicadores de la presencia de las manifestaciones de la cultura de paz en los libros de textos estudiados.

- Cambio social y participación activa: incluye la adquisición de valores a través de actitudes y comportamientos propicios para el fomento de la cultura de paz, destacando la participación activa y comprometida como elemento fundamental para la transformación social.

- Igualdad y dignidad: hace referencia a la importancia del respeto a la vida y la dignidad de cada persona, eliminando actitudes prejuiciosas, discriminatorias y promocionando la igualdad de oportunidades de todas las personas.

- Promoción de los derechos humanos y la democracia: consiste en el pleno respeto y desarrollo de los derechos humanos y de la democracia participativa.

- Promoción del desarrollo: recoge la contribución del desarrollo de todas las personas y de todos los pueblos, a través del desarrollo sostenible.

- Protección ambiental: relativo a la promoción de la conciencia ambiental, a través de un consumo responsable y un desarrollo orientado al respeto de todas las formas de vida, con el fin de poder garantizar las necesidades de desarrollo de las generaciones venideras.

- Resolución pacífica de los conflictos: la práctica de la noviolencia activa, rechazando todo tipo de violencia y comprometiéndonos con la resolución pacífica de los conflictos, entendiéndolo desde la regulación y prevención pacífica ${ }^{8}$ de los mismos.

Para la valoración (tabla 4) de la presencia y el tratamiento de estos indicadores hemos combinado la interacción de dos variables, la profundidad 
y la extensión, con tres grados para cada una: ausente, superficial y profundo, para la primera; y ausente, breve y amplio, para la segunda. De esta combinación surgen los siguientes criterios:

- Ausente (A): ausencia total del ámbito correspondiente a lo largo de todo el libro.

- Superficial breve (SB): aparece pero brevemente y con carencias.

- Superficial amplio (SA): es abordado con profusión, pero con tratamiento insuficientemente fundamentado y argumentado.

- Profundo breve (PB): buena fundamentación y explicación aunque poco desarrolladas.
- Profundo amplio (PA): buena fundamentación y argumentación con buen desarrollo y explicaciones adecuadas.

\section{Selección de los libros}

Se han elegido seis manuales de Educación Secundaria Obligatoria de editoriales con presencia estatal (tabla 1$)^{9}$, que son los más utilizados en las clases de Educación para la Ciudadanía en esta etapa, según las informaciones facilitadas en diferentes librerías ${ }^{10}$ de Granada y por comprobaciones directas en varios institutos de la ciudad, contrastadas en sus respectivas páginas webs ${ }^{11}$.

TABLA 1. Manuales analizados

Editorial Año Título

\begin{tabular}{|c|c|c|}
\hline 1. Edebé & 2007 & Educación para la Ciudadanía y los Derechos Humanos: ESO \\
\hline 2. Pearson Alhambra & 2011 & $\begin{array}{l}\text { Jóvenes Ciudadan@s, Educación para la Ciudadanía y los Derechos } \\
\text { Humanos: Educación Secundaria Obligatoria }\end{array}$ \\
\hline 3. Santillana & 2011 & Educación para la Ciudadanía: ESO: Andalucía \\
\hline 4. Ediciones Octaedro & 2011 & Educación para la Ciudadanía y los Derechos Humanos: ESO \\
\hline 5. SM & 2011 & Educación ético-cívica $4^{\circ}$ ESO \\
\hline 6. Everest & 2007 & Educación para la Ciudadanía: ESO \\
\hline
\end{tabular}

En la tabla 2 se detallan las unidades temáticas de cada libro.

TABLA 2. Unidades temáticas

Libros Índices de contenidos

1. Soy persona

2. Vivo en sociedad

Libro 1 3. Tengo derechos y deberes

4. Soy demócrata

5. En una sociedad plural

6. En un mundo global

1. Del yo al nosotros

Libro 2 2. Los derechos humanos

3. Sociedades democráticas del siglo XXI

4. La España democrática 


\section{TABLA 2. Unidades temáticas (cont.)}

Libros Índices de contenidos

5. Participación ciudadana

6. La lucha contra la discriminación

Libro 2 7. Desarrollo sostenible y consumo responsable

8. Globalización

9. La pobreza

1. Aprendemos a ser ciudadanos

2. La convivencia en el centro escolar

3. Las relaciones humanas

4. Una sociedad plural

Libro 3 5. La igualdad entre hombres y mujeres

6. La política y el bien común

7. Consumo y desarrollo sostenible

8. La convivencia ciudadana

9. Ayuda al desarrollo

10. Construyendo la paz

1. Las relaciones interpersonales: sexualidad y afectividad

2. La homosexualidad

3. Los discapacitados

4. La diversidad cultural

Libro 4 5. La conquista de los derechos de la mujer

6. La democracia

7. El consumo

8. La educación vial

9. El problema Norte-Sur

1. La condición del ser humano

2. Yo decido

3. El origen de las morales

4. ¿Qué es lo bueno?

Libro 5 5. Los derechos humanos como teoría ética

6. La política y los derechos humanos

7. El derecho, la ética y la justicia

8. La economía y los grandes problemas mundiales

9. La situación de la mujer en el mundo

1. ¡Cuenta conmigo!, me reconozco... les reconozco

2. ¿Qué gran tarea!, somos personas con valor y dignidad

3. ¡Y que cumplas muchos más!, convivencia desde el cariño y el diálogo

4. Tienes derecho, responsabilizate, derechos y deberes

Libro 6 5. Derecho a los derechos, los derechos humanos

6. Escalando igualdad, la conquista de los derechos de las mujeres

7. Vivo en una sociedad estructurada, me reconozco... les reconozco

8. Vivo en una sociedad plural, aprendo a respetar y asumo mis responsabilidades como ciudadano

9. Vivo en una sociedad plural, me muevo para mejorar el mundo

Hemos analizado la estructura de la presentación, exposición y desarrollo de los contenidos en los manuales estudiados. En la tabla 3 aparecen en I (inicial) aquellos contenidos expuestos en la presentación expositiva del libro; en D (desarrollo), los temas y las actividades destinadas a la fase de progreso del bloque o unidad; y en C (conclusión), los correspondientes a la parte final y de síntesis de los contenidos de la unidad. 


\section{Tabla 3. Estructura de los contenidos}

Libros

Contenidos por unidad

Libro 1

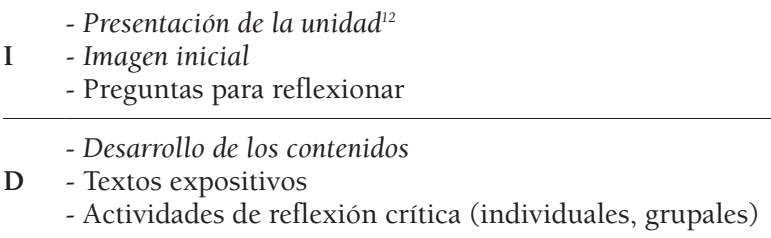

- Estrategias para el desarrollo de la inteligencia emocional

- Síntesis de la unidad

C - Exposición de recursos bibliográficos, filmográficos, webgrafía, juegos

- Actividades para la reflexión final

- Evaluación

- Anexos

I - Exploración inicial

- Actividades de iniciación

D - Desarrollo de los contenidos

- Actividades (individuales, grupales)

- Y tú... ¿qué haces? (texto final de reflexión)

C - Actividades finales

- Evaluación

I - Doble página de presentación

- Texto explicativo

Libro 3 D - Para tu reflexión (textos para profundizar)

- Debate (a partir de un texto explicativo)

C - Solidarios (muestra distintas iniciativas sociales y solidarias)

- Construir mi visión personal (testimonios y lecturas para la reflexión)

I - Presentación del problema ético

- Actividades de iniciación (individuales, grupales)

- La memoria histórica (exposición del tema a desarrollar)

Libro 4 D - Valor añadido (se trabaja un valor concreto)

- Educación de la salud y de las emociones

- Autoevaluación

C - Seguimos trabajando (exploración del tema a partir de la propuesta de una lectura, webs, película, canción)

I - Imagen y texto de presentación

- Activando la red de la memoria (establece relaciones con otras materias estudiadas)

- Exposición de contenidos

D - Actividades individuales

Libro 5

- Grandes problemas... (se presentan problemas de la humanidad y sus respuestas malas y buenas, así como la gran solución recogida en la Declaración de los Derechos Humanos)

- Debate (a partir de un texto explicativo)

- Actividades finales

C - Creadores sociales (muestra de personajes que han contribuido a la mejora de la sociedad)

- Resumen final

- Para ver y leer (exposición de bibliografía y filmografía) 
Tabla 3. Estructura de los contenidos (cont.)

Libros Contenidos por unidad

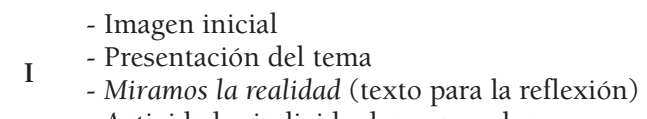

Libro 6

- Actividades individuales y grupales

D - Desarrollo de contenidos

- Actividades individuales y grupales

C - Ya sabes, ya puedes hacer (propuesta de webs, lecturas, cine y música, relacionadas con el tema)

C - Prueba con... aprueba con... (actividades de autoevaluación)

\section{Resultados}

En la tabla 4 se expone un resumen de los resultados obtenidos, organizados en función de la profundidad y la extensión de la contribución de los contenidos de cada uno de los libros estudiados a los respectivos ámbitos de la cultura de paz utilizados como indicadores. Siguiendo las denominaciones señaladas, A se corresponde con ausencia de ambas variables, SB con superficial breve, SA con superficial amplio, PB con profundo breve y PA con profundo amplio. Esta escala cualitativa podría ser cuantificada de 1 a 5 siendo 1 "ausente" y 5 "profundo amplio".

Veamos el tratamiento que se hace en los libros sobre los seis ámbitos establecidos como indicadores.

\section{Cambio social y participación activa}

Hemos encontrado que este ámbito es tratado en los seis libros con amplitud y profundidad, de manera muy comprometida con la construcción de la cultura de paz, siendo el único en el que prevalecen las dos variables en todos los manuales estudiados. Encontramos propuestas muy favorables para el cambio social ligado a una participación activa y responsable, capaz de favorecer y fortalecer el cumplimiento de los derechos humanos y el desarrollo de la cultura de paz. Nuestros resultados no coinciden con los de Boqué, Alguacil, Pañellas y García Raga (2014), que en su análisis de los libros de primaria detectan escasez de ejemplos a la hora de incentivar y trabajar la participación ciudadana.

Tabla 4. Resumen de los resultados

\begin{tabular}{lllllll} 
& \multicolumn{3}{c}{ Profundidad y extensión de su tratamiento en cada libro } \\
\cline { 2 - 7 } Indicadores & 1 & 2 & 3 & 4 & 5 & 6 \\
\hline Cambio social y participación activa & PA & PA & PA & PA & PA & PA \\
\hline Igualdad y dignidad & SB & PA & PA & PA & PA & PA \\
\hline Promoción de los derechos humanos y la democracia & SA & SA & PA & SA & PA & PA \\
\hline Promoción del desarrollo & SB & PA & PA & PA & PB & PA \\
\hline Protección ambiental & SB & PA & PA & SB & SB & SB \\
\hline Resolución pacífica de los conflictos & PA & SB & PA & SB & PA & PB \\
\hline
\end{tabular}


Destaca el importante papel que se otorga a la educación emocional y las habilidades sociales como herramientas de trasformación y cambio social en los libros $1,2,3,5$ y 6 , poniendo de manifiesto que conocer, expresar y controlar nuestras emociones nos servirá para entender también las de los demás, y que el equilibrio emocional resulta imprescindible para aprender a convivir en armonía, lo que se considera fundamental para el cambio social. Por su parte, el libro 4 da mayor importancia a la participación activa y comprometida de la ciudadanía como motor de transformación social.

Se da una especial trascendencia a la empatía, trabajada en todos los libros como cualidad esencial para ser aprendida en todos los ámbitos de la vida, sirviéndonos como revulsivo frente a la discriminación y la violencia.

Aparece el cuidado como valor fundamental para construir la cultura de paz, sobre todo en los libros 2 y 5 . Concretamente el libro 5 pone de relieve expresamente la necesidad de extender y fomentar una "cultura del cuidado". En el resto de libros se presta también una importante atención a la esfera del afecto, pero de manera más general.

El binomio libertad y responsabilidad aparece desarrollado en las seis editoriales. Se ofrece la visión generalizada de que para una adecuada relación con los demás, la libertad debe ir de la mano de la responsabilidad, teniendo presente que no estamos solos y que nuestros actos afectan a todo lo que nos rodea. También es defendida en todos los libros la obligación ética y moral de ayudar y ofrecer oportunidades a las personas más necesitadas. De igual modo, se aprecia en todos los manuales la urgencia de fomentar en la juventud el desarrollo del espíritu crítico para una participación responsable en la sociedad.

En cuanto a la implicación de los centros educativos en los casos de maltrato y acoso escolar, encontramos que los libros 2 y 4 trabajan esta temática de forma profunda y comprometida, instando a la juventud a que sea activa, valiente y solidaria, denunciando los casos y prestando ayuda a las víctimas.

La importancia del voluntariado se recoge en todos los manuales, pero es en los libros 2 y 5 donde se potencia especialmente el papel de jóvenes anónimos que trabajan activamente por la solidaridad, fomentándose desde esta perspectiva el empoderamiento de la juventud y la creación de capital social.

\section{Igualdad y dignidad}

En este ámbito es muy notable la importancia y el compromiso que plantean todos los autores con la igualdad y la dignidad de las personas, desde la perspectiva de la igualdad en la diversidad. Excepto en el libro 1, que ofrece un tratamiento más superficial y breve de este indicador, en todos los demás se trabaja con profundidad y amplitud.

Todos los libros encaran este tema con gran implicación ética. En la totalidad de los manuales se recuerda a las personas que actualmente y a lo largo de la historia han sido y son señaladas y estigmatizadas por ser consideradas diferentes al patrón hegemónico centrado en el patriarcado occidental, tales como mujeres, homosexuales, personas con diversidad funcional, minorías étnicas o practicantes de religiones distintas a la católica; al igual que las personas sin recursos económicos, refugiados, inmigrantes, etc. Todas las editoriales aportan evidencias de cómo todos estos colectivos han sido marginados y rechazados a lo largo de la historia. Solo en el libro 1 se realiza una aproximación con menor detalle y más centrada en las diferencias que generan la construcción de prejuicios y estereotipos.

El mayor compromiso con el respeto a la homosexualidad lo desarrollan los libros 2, 3 y 4. 
Estos dos últimos nos acercan a la realidad a través de los distintos tipos de familias que están surgiendo en la actualidad, gracias a las políticas de igualdad y a las vindicaciones de numerosos pensadores y grupos sociales.

La diversidad funcional aparece muy adecuadamente tratada en los libros 4, 5 y 6 . Se pone de manifiesto la obligación de la ciudadanía a la atención y el cuidado de los más vulnerables, así como la necesidad de la colaboración de todos para hacerles la vida más fácil, cediéndoles poder para facilitar su plena participación en la sociedad.

Todos los libros ensalzan muy positivamente las contribuciones realizadas por los movimientos sufragistas y por el feminismo. Destaca el libro 4 por ser el único que realiza un enfoque de género en el lenguaje a lo largo de todo el texto.

La totalidad de los libros denuncian los problemas que plantea la construcción de prejuicios y estereotipos capaces de generar comportamientos hostiles y predisposiciones de recelo con la alteridad que desencadenen actitudes de rechazo y violencia.

En las seis editoriales se enfatizan las personalidades, los movimientos y grupos sociales que han luchado por la equidad, la dignidad, la justicia y la paz a través de la historia. En el libro 5 nos encontramos con una original representación dentro del apartado "creadores sociales" donde ensalza la figura de adolescentes anónimos que han destacado por haber desarrollado una importante obra social.

El papel de las mujeres como constructoras de paz, más allá de ser visibilizadas solo como víctimas que sufren, se explica en los libros 1 , $3,4,5$ y 6 , aunque también se evidencie la precariedad y la desigualdad que sufren las mujeres y niñas en la actualidad, así como la feminización de la pobreza. En estos libros se trasciende esta visión argumentando que el desarrollo del derecho a la igualdad pasa por el empoderamiento femenino.

\section{Promoción de los derechos humanos y la democracia}

En este ámbito los libros 1, 2 y 4 mantienen un nivel superficial; aunque los tres aportan un extenso tratamiento, no pasan de la mera descripción. Los otros tres sí lo afrontan en profundidad y con la extensión adecuada.

Destaca considerablemente el cumplimiento y respeto de los derechos humanos y de la democracia en los seis libros analizados. La diferencia estriba en el modo en que se trabajan estos aspectos. Los que lo hacen de forma superficial y amplia desarrollan la evolución de las temáticas de forma estereotipada, con actitud poco crítica y repitiendo los mismos hitos, datos y personalidades, resultando menos atractivos para fomentar la reflexión del alumnado. Coincidimos con Ortega, Tejedor y Ruiz (2012), que en su estudio constatan que los derechos humanos son tratados en todos los manuales revisados, aunque también encuentran en buena parte de ellos una "falta de visión crítica que pueda generar una reflexión por parte del alumnado y tomar postura e identidad respecto a tales cuestiones" (p. 74).

El libro 5 aborda la evolución de los derechos humanos, especificando los de primera, segunda y tercera generación, destacando en esta última el tratamiento del "derecho a la paz", al ser el único libro que lo recoge, a pesar de ser uno de los más importantes de esta última generación.

Todos los libros denuncian la situación de injusticia que supone "cerrar las puertas" a los inmigrantes sin documentación y a los refugiados, denunciando el incumplimiento por parte de muchos Estados de garantizar los derechos de asilo y de acogida como derechos humanos. También todas las editoriales muestran gran preocupación por la igualdad y los derechos de las mujeres.

\section{Promoción del desarrollo}

Este ámbito se desarrolla con bastante profundidad. Solamente en el libro 1 es trabajado 
de manera más breve y superficial, con temáticas interesantes pero sin analizar las relaciones de unos aspectos con otros ni detenerse suficientemente en el tratamiento de cada uno de ellos.

El objetivo de la erradicación de la pobreza es un tema común, al igual que la denuncia de las consecuencias de la globalización. Los seis libros señalan el papel de los países ricos como principales responsables de la falta de desarrollo de los países desfavorecidos a causa de sus prácticas poco éticas, así como de las trabas que les ponen para que puedan alcanzar cotas de desarrollo aceptables para la dignidad humana. Los libros 3, 4 y 5 ofrecen posibles soluciones a esta compleja situación, presentando los problemas mundiales desde una perspectiva crítica e invitando al alumnado a responder a pequeña escala desde su contexto más próximo, concretado en el hogar, el barrio y la escuela.

Los libros 2, 3, 4 y 6 enriquecen este ámbito con mayor amplitud. El libro 5 lo realiza más brevemente, pero resulta de gran interés que manifieste la importancia del fomento y desarrollo de una cultura de paz para abordar la problemática al desarrollo: "Las organizaciones internacionales pretenden crear una cultura de paz, de solidaridad y de justicia: este es el proyecto común de la humanidad. Por eso, es necesario promover una cultura ética, que defienda los derechos y los deberes" (Marina, 2011: 145). Llama la atención negativamente que apenas se trabaje explícitamente la cultura de paz en los manuales, tanto en su versión original de Naciones Unidas como en otras acepciones más amplias, por lo que resulta muy poco probable, como indican Boqué et al. (2014), que sea tratada durante el desarrollo curricular de otras materias.

\section{Protección ambiental}

En este ámbito encontramos dos posturas opuestas. En los libros 1, 4, 5 y 6 se trata con brevedad y bastantes carencias, mientras que en los otros se hace de un modo más participativo y con mayor información y profundidad. Coincidimos, por tanto, con Boqué et al. (2014) en que el desarrollo de la conciencia ambiental no tiene suficiente presencia en los libros de texto, a pesar de ser uno de los elementos fundamentales para el sostenimiento de la paz. Y tanto la paz positiva como negativa no pueden lograrse sin seguridad ambiental y responsabilidad ecológica (Bajaj y Chiu, 2009).

El leitmotiv de los seis libros es la crítica al consumismo, al despilfarro, la irresponsabilidad de la ciudadanía y especialmente la de los países ricos, planteando como posible solución el compromiso de todos con la realización de actividades, comportamientos y políticas encaminadas a un desarrollo sostenible que pueda satisfacer las necesidades de todos los seres vivos.

En los libros 2 y 3 se trata con profundidad, exponiendo posibles soluciones a estos problemas y recordando que los pequeños detalles que todos podamos aportar pueden ayudar bastante a la protección medioambiental. También nos encontramos en estos dos libros con una gran implicación en plantear la interdependencia de todos los seres vivos y la importancia de que el ser humano entienda que es él el que pertenece a la tierra y no al contrario. En el libro 3 se promueve el activismo de la ciudadanía en la protección medioambiental insistiendo en que esta labor no es solo responsabilidad de las políticas internacionales.

\section{Resolución pacífica de los conflictos}

Este último ámbito se trabaja de manera profunda y comprometida en los libros $1,3,5$ y 6 , cuyos tratamientos resultan muy próximos y favorables al afrontamiento positivo de los conflictos, destacando el papel que juegan especialmente los procesos y técnicas de mediación, el aprendizaje de habilidades sociales y la inteligencia emocional. En los otros dos libros estos aspectos son tratados de manera concisa y superficial, con un tratamiento escaso e indirecto. 
La educación emocional como herramienta para la regulación y prevención de los conflictos es trabajada en los libros $1,2,3,5$ y 6 , señalándose la vital importancia de contar con una adecuada educación de los sentimientos para poder relacionarnos pacíficamente con los demás, empezando desde el amor a uno mismo para poder extrapolarlo a los demás.

El libro 1 se centra en explicar los distintos tipos de violencia y los diferentes conflictos que se pueden producir, dedicando un apartado a aprender cómo solucionarlos. En relación con ello se ofrece como referente la definición de paz de la $\mathrm{ONU}^{13}$. Es destacable que solamente los libros 1, 3 y 5 ensalzan la paz como forma de vida, como modo de pensar, actuar y vivir desde el que, a través de la resolución pacífica de los conflictos y de sus técnicas, podamos aprender a solucionar nuestros problemas sin que degeneren en situaciones violentas.

El libro 3 resalta la importancia de afrontar adecuadamente los conflictos para que no deriven en situaciones tan preocupantes en la actualidad como el acoso escolar, siendo el manual que está más centrado en el aprendizaje de la resolución de conflictos en el ámbito de la escuela. Por su parte, el 5 señala como objetivo prioritario la visibilización de la paz desde la cotidianeidad, siendo el único que explícitamente aborda el concepto de construcción de paz.

Destaca la importancia que se le da a la mediación como vía para la trasformación pacífica de los conflictos, especialmente en los libros 1 y 5 , mientras que el 6 se centra más en la importancia del diálogo, la educación emocional y la comunicación no verbal.

\section{Discusión y conclusiones}

Relacionando los resultados obtenidos con los objetivos de este trabajo, encontramos en el análisis realizado valiosas muestras para visibilizar, construir y mantener una cultura de la paz. De hecho, uno de los primeros aspectos que podemos destacar es que la valoración de "ausente" para las dos variables utilizadas, profundidad y extensión, no se da en ninguna de las editoriales. Igualmente contamos con interesantes propuestas de las editoriales para facilitar los aprendizajes que ayuden a desarrollar actitudes orientadas al fomento de la paz. Con carácter general, también se aprecia cierto equilibrio a la hora de abordar las temáticas, de manera que si un libro presenta varias dimensiones trabajadas deficientemente, suele compensarlo aportando mayor énfasis en el tratamiento de otras.

Destacamos que el ámbito cambio social y participación activa ha sido trabajado con mayor compromiso para el desarrollo de la cultura de la paz en los seis libros, mientras que el de protección ambiental es sobre el que se aporta menor información, detalle y profundidad.

En la totalidad de los libros de texto analizados, además de denunciar la violencia existente, se aportan posibles soluciones desde la cotidianeidad y los pequeños detalles, poniendo de manifiesto que en manos de todos y cada uno de nosotros está transformar los espacios violentos en lugares de convivencia pacífica, como nos recuerda la filosofía para hacer las paces que propone Martínez Guzmán (2001).

Sobre la influencia que para nuestro estudio tiene la asignatura de Educación para la Ciudadanía, con repercusiones pedagógicas más importantes que las disputas entre sectores políticos y sociales, que en muchas ocasiones han olvidado su valor educativo, hemos podido comprobar que su desaparición supondría un importante obstáculo para el fomento de valores cívicos fundamentales relacionados con los ámbitos de contenidos tratados en nuestro trabajo. Aunque, como hemos señalado, en el curso actual se siguen utilizando los mismos libros promovidos por la anterior ley de educación (LOE), no debemos olvidar que el propósito de la actual ley (LOMCE) es su sustitución por la asignatura de 
Valores Éticos, lo que supone la generación de nuevos materiales con perspectivas distintas a las actuales.

En nuestro estudio hemos encontrado una alta coherencia y estrecha relación entre los ámbitos analizados en los seis manuales y los contenidos y competencias propuestas en el Real Decreto por el que se regula la asignatura de Educación para la Ciudadanía ${ }^{14}$. En primer lugar encontramos que en el bloque 1 figuran los contenidos comunes para los cuatro cursos, con temáticas encaminadas a desarrollar aquellas habilidades y destrezas relacionadas con la reflexión y con la participación ${ }^{15}$, lo que conecta claramente con el cambio social y participación activa.

Estrechamente relacionados con la igualdad y la dignidad encontramos el bloque 2, Relaciones interpersonales y participación, en uno de los tres primeros cursos; y en el bloque 2, Identidad $y$ alteridad, y el bloque 6, La igualdad entre hombres y mujeres, de cuarto curso.

Los contenidos que guardan una completa relación con el ámbito de la promoción de los derechos humanos y la democracia los encontramos en el bloque 3, Deberes y derechos ciudadanos, y en el bloque 4, Las sociedades democráticas del siglo XXI, en uno de los tres primeros cursos; así como en los bloques 3, Ética y política, y 4, Teorías éticas, los derechos humanos, de cuarto.
Finalizando con el bloque 5, en uno de los tres primeros cursos, Ciudadanía en un mundo global, y el bloque 5 de cuarto curso, Problemas sociales del mundo actual, que desarrollan aspectos y contenidos conectados íntimamente con los ámbitos promoción del desarrollo, resolución pacífica de los conflictos, y, de menor manera, con la dimensión de protección ambiental.

A lo largo del Real Decreto que regula la asignatura también se aprecia la implicación con la construcción de la cultura de la paz, recogiéndose expresamente en varias ocasiones la importancia de fomentarla y valorarla.

Igualmente hemos analizado el Real Decreto que regula la asignatura de Valores Éticos ${ }^{16}$, donde podemos observar un cambio sustancial en las temáticas propuestas y en la perspectiva de enfoque, prevaleciendo un tratamiento meramente descriptivo y acrítico, alejado de análisis orientados a la transformación y el empoderamiento social, eludiendo temas muy importantes relacionados con la igualdad y la dignidad de todas las personas.

Por ello consideramos imprescindible mantener la Educación para la Ciudadanía, especialmente en el currículo de Educación Secundaria Obligatoria, entendiendo, como apunta Bolívar (2007), que esta formación no puede circunscribirse solo al ámbito escolar, sino que es preciso extenderla a otras esferas de socialización.

\section{Notas}

${ }^{1}$ Pactos de 1966 y Derechos Humanos de la "Tercera Generación".

${ }^{2}$ El Derecho Humano a la Paz. Declaración del Director General de la UNESCO. París, Francia. Enero, 1997.

${ }^{3}$ Resolución 53/243, de 13 de septiembre de 1999, Declaración y Programa de Acción sobre una Cultura de Paz. Asamblea General de las Naciones Unidas.

${ }^{4}$ Recomendación [Rec (2002)12] del Comité de Ministros a los Estados miembros sobre la educación para la ciudadanía democrática adoptada por el Comité de Ministros el 16 de octubre de 2002.

${ }^{5}$ Real Decreto 1631/2006, de 29 de diciembre, por el que se establecen las enseñanzas mínimas correspondientes a la Educación Secundaria Obligatoria (BOE, nº 5, de 5/1/2007).

${ }^{6}$ Véase la nota 3.

${ }^{7}$ Véase el Manifiesto 2000 para una cultura de paz y no violencia. 
${ }^{8}$ Véase Muñoz, Francisco A. (2004). Regulación y prevención de conflictos, en B. Molina y F. A. Muñoz, Manual de Paz y Conflictos (pp. 171-200). Universidad de Granada: Eirene.

${ }^{9}$ La información bibliográfica completa se ofrece en las referencias.

${ }^{10}$ Las librerías consultadas han sido Picasso, Babel y Teorema.

${ }^{11}$ Véanse en la webgrafía las páginas de los institutos Alba Longa, Arabuleila, Blas Infante, Federico García Lorca, Fernando de los Ríos, Ilíberis y Luis Bueno Crespo.

${ }^{12}$ Mantenemos en cursiva los títulos originales de los correspondientes epígrafes y apartados recogidos en los libros.

${ }^{13}$ Véase la nota 1.

${ }^{14}$ Véase la nota 5.

${ }^{15}$ En cursiva mantenemos la redacción original del texto del Real Decreto.

${ }^{16}$ Real Decreto 1105/2014, de 26 de diciembre, por el que se establece el currículo básico de la Educación Secundaria Obligatoria y del Bachillerato (BOE, no 3, de 3/1/2015).

\section{Referencias bibliográficas}

Aguilar, T., Caballero, A., Dausà, N., Mestre, J. V., y Vilaseca, S. (2007). Educación para la Ciudadanía y los Derechos Humanos: ESO. Barcelona: Edebé.

Aran, J. M., Güell, M., Marías, I., y Muñoz, J. (2011). Educación para la Ciudadanía y los Derechos Humanos: ESO. Barcelona: Ediciones Octaedro.

Bajaj, M., y Chiu, B. (2009). Education for Sustainable Development as Peace Education. Peace $\mathcal{E}$ Change, 34(4), 441-455.

Bellido, L. J., García Martín, S., y García Peña, J. L. (2007). Educación para la Ciudadanía: ESO. León: Proyecto Equalia, Everest.

Bolívar, A. (2007). Educación para la Ciudadanía. Algo más que una asignatura. Barcelona: Graó.

Boqué, M. C., Alguacil, M., Pañellas, M., García Raga, L., y Rosich, C. (2013). Medir la Paz. Indicadores de construcción de la Cultura de Paz a través de la Educación. En C. Martínez López y S. Sánchez Fernández (eds.), Escuela, espacio de paz: experiencias desde Andalucía (pp. 191-234). Granada: Universidad de Granada, colección Eirene.

Boqué, M. C., Alguacil, M., Pañellas, M., y García Raga, L. (2014). La cultura de paz en la educación para la ciudadanía y los derechos humanos en los libros de texto de educación primaria. Perfiles Educativos, XXXVI, 146, 80-97.

De Rivera, J. (2004). Assessing the Basis for a Culture of Peace in Contemporary Societies. Journal of Peace Research, 41(5), 531-548. doi: 10.1177/0022343304045974

García López, R., y Murillo, J. (2009). Las referencias a la Educación para la Ciudadanía en la prensa. Bordón. Revista de Pedagogía, 61 (1), 77-93.

Marina, J. A. (2011). Educación ético-cívica $4^{\circ}$ ESO. Madrid: Grupo SM.

Martínez Guzmán, V. (2000). Saber hacer las Paces. Epistemologías de los Estudios para la Paz. Convergencia, 23, 49-96.

Martínez Guzmán, V. (2001). Filosofía para hacer las paces. Barcelona: Icaria.

Muñoz, F. A. (ed.) (2001). La Paz Imperfecta. Granada: Universidad de Granada, colección Eirene.

Muñoz, F. A. (2004). Regulación y prevención de conflictos. En B. Molina y F. A. Muñoz (eds.), Manual de Paz y Conflictos (pp. 171-200). Granada: Universidad de Granada.

Ortega, S., Tejedor, M., y Ruiz, E. (2012). Análisis de la Educación para el Desarrollo en los manuales de Educación para la ciudadanía de Educación Secundaria Obligatoria. REIFOP, 15(2), 71-78.

Pellicer, C., y Ortega, M. (2011). Educación para la Ciudadanía: ESO: Andalucía. Madrid: Santillana, Proyecto La Casa del Saber. 
Pérez Carrasco, J., Díaz Otero, C., y Díaz Fleitas, J. M. (2011).Jóvenes Ciudadan@s, Educación para la ciudadanía y los derechos humanos: Educación Secundaria Obligatoria. Madrid: Pearson Alhambra.

Sánchez Fernández, S., y Sánchez Vázquez, A. (2012). La convivencia escolar desde la perspectiva de la Cultura de Paz. Convives, 0, 36-41.

UNESCO (1996). From a culture of violence to a culture of peace. París: Unesco.

Webgrafía

IES Alba Longa. Recuperado de http://www.juntadeandalucia.es/averroes/centros-tic/18700232/helvia/sitio/index.cgi?wid_seccion=8\&wid_item $=53$

IES Arabuleila. Recuperado de http://www.juntadeandalucia.es/averroes/centros-tic/18003004/helvia/sitio/

IES Blas Infante. Recuperado de http://iesblasinfanteogijares.es/

IES Federico García Lorca. Recuperado de http://iesfgl.es/

IES Fernando de los Ríos. Recuperado de http://iesfernandodelosrios.es/

IES Ilíberis. Recuperado de http://iesiliberis.com/ies/es/

IES Luis Bueno Crespo. Recuperado de http://www.iesluisbuenocrespo.es/

UNESCO. El Derecho Humano a la Paz. Declaración del Director General. Recuperado de http://www. unesco.org/cpp/sp/declaraciones/HRtoPeace.htm

UNESCO. Manifiesto 2000 para una cultura de paz y no violencia. Recuperado de http://www3. unesco. org/manifesto2000/default.asp

\section{Abstract}

Teaching the culture of peace at the level of Compulsory Secondary Educational. A comparative study of textbooks on Citizenship Education

INTRODUCTION. The main aim of this research is to understand and analyze the teaching approaches that exist of the contents which are related to the culture of peace education in the textbooks on Citizenship Education in Compulsory Secondary Education. As this subject is the closest to these contents. METHOD. We have reviewed the status of the issue, and from a qualitative approach we have analyzed six textbooks on Citizenship Education in Compulsory Secondary Education. Each book has been examined using six parameters, keeping in mind which of these are based on the main documents that explain and develop the culture of peace. To evaluate the existence and treatment of these parameters, we have established as our criteria the combination of two variables; the degree of depth and development with three assessment scales for each one: Non-existent, superficial and profound for the first; and nonexistent, brief and broad for the second. RESULTS. We have found that most of the analyzed textbooks contain and develop in a suitable way almost all the content reflected in the parameters that we have used. In addition, they all condemn ongoing violence and propose building a culture of peace at different levels and in different fields, from the international to the day to day level. DISCUSSION. In contrasting our results with other studies and legislative documents on education, we have seen that the demise of the subject of Citizenship Education represents a significant obstacle to training in core values associated with the culture of peace, which are especially important at this educational stage of development.

Keywords: Peace, Citizenship Education, Compulsory Secondary Education Schools, Textbooks, Values Education. 


\section{Résumé}

La culture de la paix dans l'enseignement secondaire. Étude comparative des manuels d'éducation à la citoyenneté

INTRODUCTION. Cette recherche a pour objectif principal de connaître et d'analyser la présence et le traitement didactique du contenu lié à l'éducation pour la culture de la paix dans les manuels d'éducation à la citoyenneté dans l'enseignement secondaire où la paix fais souvent l'objet des programmes. METHODE. Analysées les études précédentes les plus représentatifs, à partir d'une approche qualitative nous avons analysé six manuels de la matière «éducation à la citoyenneté» dans l'enseignement secondaire. Chaque ouvrage a été étudié à travers six indicateurs élaborés tenant en compte des principaux documents qui expliquent et développent la culture de la paix. Pour l'évaluation de la présence du traitement de ces indicateurs, nous avons établi les critères par la combinaison de deux variables, la profondeur et la largeur des contenus, qui comportent chacune trois degrés d'évaluation: absentes, superficielles et profondes pour la première ; absente, courte et large pour la seconde. RÉSULTATS. Nous avons constaté que la plupart des manuels analysés développant suffisamment la question fournissent d'information en relation aux indicateurs utilisés. Également, ils dénoncent la violence existante et posent des alternatives pour construire une culture de la paix à différents niveaux et dans des domaines variés, comprenant l'international et le plus local et proche. DISCUSSION. En comparant nos résultats avec d'autres études ainsi qu'avec des documents législatifs sur l'éducation, nous avons constaté que la disparition de la matière "éducation à la citoyenneté" représente un frein indéniablement à la formation aux valeurs fondamentales qui sont liées à la culture de la paix et dont le traitement est spécialement importante au cours de cette étape éducative.

Mots-clés: Paix, Éducation à la citoyenneté, L'enseignement secondaire, Les manuels, Léducation des valeurs.

\section{Perfil profesional de los autores}

\section{Sebastián Sánchez Fernández (autor de contacto)}

Catedrático de Didáctica y Organización Escolar e investigador del Instituto Universitario de Investigación de la Paz y los Conflictos de la Universidad de Granada. Coordinador del Máster Universitario en "Diversidad Cultural. Un enfoque multidisciplinar y transfronterizo". La docencia e investigación la desarrolla fundamentalmente en educación para la cultura de paz y en educación intercultural en el Campus de Melilla de esta universidad.

Correo electrónico de contacto: ssanchez@ugr.es

Dirección para la correspondencia: Departamento de Didáctica y Organización Escolar. Facultad de Educación y Humanidades. Campus Universitario, C/ Santander no 1. 52005 Melilla. E Instituto Universitario de Investigación de la Paz y los Conflictos, C/ Rector López Argüeta, s/n. 18071 Granada. 


\section{Miriam Vargas Sánchez}

Miriam Vargas Sánchez, doctoranda en el Programa de Doctorado en Estudios de las Mujeres, Discursos y Prácticas de Género. Diplomada en Educación Social por la Universidad de Granada. Máster oficial en "Cultura de Paz, Conflictos, Educación y Derechos Humanos" por la Universidad de Granada. Máster propio en "Mediación Familiar, Social y Laboral" por la Universidad de Granada. Correo electrónico de contacto: mirivs@outlook.com 